\title{
LOS/NLOS Detection for UWB Signals: A Comparative Study Using Experimental Data
}

\author{
Nicolò Decarli, \\ Davide Dardari \\ WiLAB, DEIS, \\ University of Bologna at Cesena, \\ via Venezia 52, 47023 Cesena (FC), Italy \\ Email: \{ndecarli, ddardari\}@ieee.org
}

\author{
(Invited Paper)
}

\author{
Antonio Alberto D'Amico \\ Department of Information Engineering \\ of the University of Pisa, \\ Via Caruso 16, Pisa, Italy \\ Email: antonio.damico@iet.unipi.it
}

\begin{abstract}
In this paper the problem of detecting the channel state between LOS and NLOS conditions is addressed using UWB signals. A new distribution-based identification approach is proposed and its performance is compared with that of other classic schemes. To this purpose experimental data collected in realistic environments have been used.
\end{abstract}

\section{INTRODUCTION}

Ultrawide bandwidth (UWB) indoor localization is an important technology for use in a variety of civil and military application [1], [2]. In the commercial world, for example, there is an increasing demand for identifying the location of specific items in warehouses and cargo ships. Similarly, in public safety applications, it is important to implement devices for guiding policemen and fire-fighters inside buildings during their missions.

Localization systems can provide location estimates through range measurements. A variety of techniques based on timeof-arrival (TOA), angle-of-arrival (AOA), time difference-ofarrival (TDOA) and received signal strength (RSS) are available to perform range measurements in an indoor environment [3]. In the following, we concentrate on TOA-based UWB ranging, where the distance between the mobile station (MS), or agent, and the base stations (BSs), or beacons, is estimated as the product of the signal propagation delay and the speed of light.

As studied in the literature [2], [4], [5], the performance of TOA-based ranging depends critically on the presence of a line-of-sight (LOS) channel between the MS and the base station (BS). Three BSs are sufficient to accurately solve a two-dimensional localization problem if their links to the MS are all in LOS. Unfortunately such a condition seldom holds in typical indoor applications, in which the direct path (DP) is likely to be obstructed by obstacles such as walls. The absence of a direct path results in an extra-delay for the time-of-flight (TOF) and a consequent biased estimation of the real distance [6], [7]. The knowledge of channel obstructions may improve

This research was supported by the European Commission in the framework of the FP7 Network of Excellence in Wireless COMmunications NEWCOM++ (contract no. 216715). Authors would like to thank Umberto Mengali for his suggestions in preparing this work. the accuracy of positioning system discarding non line-ofsight (NLOS) measurements or trying to correct the error downgrading ranging estimations of a certain value related to the propagation conditions of the considered scenario.

Several techniques have been proposed recently in order to identify channel conditions in terms of obstruction [8]-[19]. Obstruction detection is generally performed by extracting a certain feature from the received waveform that vary with different channel conditions. For example in [15] the identification is based on the first peak amplitude of the received signal and delay between the first and the strongest path. In [9], [10], root mean square (RMS) delay-spread, mean excess delay and kurtosis parameters are used for that purpose. The detection can also be realized without observing the received waveform directly, which is the case of the non-parametric approach proposed in [8]. This work assumes that multiple and independent TOA measurements between MS and BS are available and that the change in location of the MS during such measurements is negligible. In these conditions, the probability density function (PDF) of distance estimates between MS and BS is obtained from the measurements and is compared with the PDF corresponding to LOS propagation. If the distance between the PDFs is less than a given threshold, the channel is declared LOS, otherwise it is stated as NLOS. Another recent non-parametric solution based on least-squares support vector machines can be found in [19]. It should be noted that most of the studies in the literature evaluate the performance of the proposed schemes using certain channel models and no comparison using measured data is available to the best of authors' knowledge.

In this work some existing and new channel LOS/NLOS identification algorithms are presented and compared in a common realistic scenario using UWB measurements collected in an extensive measurement campaign.

\section{Channel State identification Algorithms}

\section{A. Classic Identification Approach}

Most of the LOS/NLOS identification techniques proposed in the literature can be summarized according to the following classic binary detection scheme, where the detection 
is performed by extracting a certain number $N$ of features $\gamma=\left\{\gamma_{1}, \gamma_{2}, \ldots, \gamma_{N}\right\}$ from the received signal and applying the classical decision theory with a likelihood ratio test (LRT):

$$
\frac{p(\gamma \mid \text { LOS })}{p(\gamma \mid \text { NLOS })} \underset{\mathcal{H}_{1}}{\stackrel{\mathcal{H}_{0}}{\gtrless}} \frac{p(\text { NLOS })}{p(\text { LOS })}
$$

where $p(\gamma \mid$ LOS $)$ and $p(\gamma \mid$ NLOS $)$ are, respectively, the joint PDFs of the set of features $\left\{\gamma_{1}, \gamma_{2}, \ldots, \gamma_{N}\right\}$ under LOS and NLOS conditions, $p(\mathrm{LOS})$ and $p(\mathrm{NLOS})$ are the prior probabilities of the LOS and NLOS events, respectively, $\mathcal{H}_{0}$ denotes the hypothesis of a LOS condition and $\mathcal{H}_{1}$ the presence of a certain obstruction.

Different techniques are then often distinguished by different choice of the set $\gamma$ of signal features. When more than one parameter is extracted from the signal, for example $\gamma_{1}$ and $\gamma_{2}$, obtaining the joint PDF can be difficult. A sub-optimal approach is to consider $\gamma_{1}$ and $\gamma_{2}$ as independent random variables. Then, the decision rule becomes

$\frac{p\left(\gamma_{1}, \gamma_{2} \mid \text { LOS }\right)}{p\left(\gamma_{1}, \gamma_{2} \mid \text { NLOS }\right)}=\frac{p\left(\gamma_{1} \mid \text { LOS }\right)}{p\left(\gamma_{1} \mid \text { NLOS }\right)} \frac{p\left(\gamma_{2} \mid \text { LOS }\right)}{p\left(\gamma_{2} \mid \text { NLOS }\right)} \underset{\mathcal{H}_{1}}{\stackrel{\mathcal{H}_{0}}{\gtrless}} \frac{p(\text { NLOS })}{p(\text { LOS })}$.

In many practical cases, nodes that are estimating their relative distance perform several consecutive measurements; hence, a large set of waveforms is usually available. In this case, we can decide for one or the other hypothesis observing the complete set of collected waveforms (assuming a quasistationary scenario), considering in (1) or (2) the average value of the parameter(s).

\section{B. Distribution-Based Identification Approach}

In this paper we propose a different method for exploiting the complete set of waveforms instead of taking decision on the single waveform. The idea is to provide an estimation of the probability distribution of the parameter of interest, and to compare it with the reference ones corresponding to LOS and NLOS propagation. The decision is taken in favor of the hypothesis for which the estimated distribution is at minimum "distance" to the reference one. The distance between distributions has to be defined according to a certain metric. Examples of such metrics are the Euclidean distance and the relative entropy or Kullback-Leibler distance. The decision criterion is then given by

$$
\frac{D\left(\widehat{p}_{\boldsymbol{\gamma}} \| p_{\boldsymbol{\gamma}}^{\text {(nlos) }}\right)}{D\left(\widehat{p}_{\boldsymbol{\gamma}} \| p_{\boldsymbol{\gamma}}^{(\text {los })}\right)} \underset{\mathcal{H}_{1}}{\stackrel{\mathcal{H}_{0}}{\gtrless}} \frac{p(\mathrm{NLOS})}{p(\mathrm{LOS})}
$$

where $\widehat{p}_{\gamma}$ denotes the estimated joint distribution while $p_{\gamma}^{\text {(los) }}$ and $p_{\gamma}^{\text {(nlos) }}$ are the reference distributions of the two hypotheses. For $N=1$ and equal prior probabilities for the two channel states, we have

$$
D\left(\widehat{p}_{\gamma} \| p_{\gamma}{ }^{(\text {nlos })}\right) \underset{\mathcal{H}_{1}}{\stackrel{\mathcal{H}_{0}}{\gtrless}} D\left(\widehat{p}_{\gamma} \| p_{\gamma}^{(\text {los })}\right) .
$$

The experimental results related to this identification method presented in section VI are obtained by using as metric

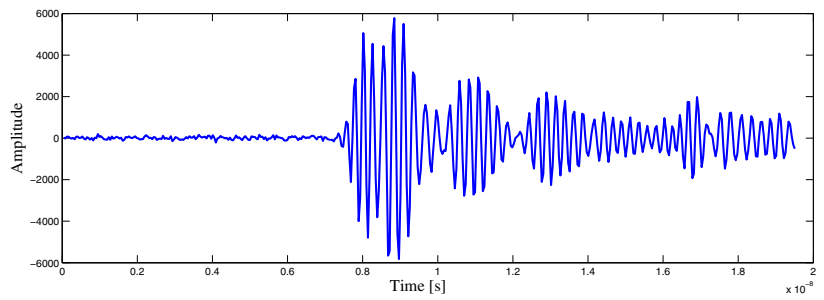

(a)

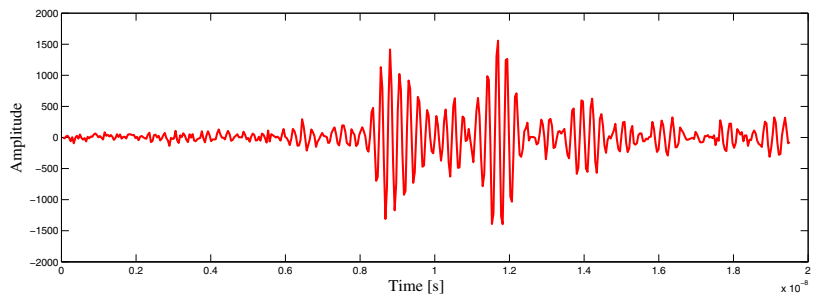

(b)

Fig. 1. Example of LOS (a) and NLOS (b) waveforms

of comparison the Euclidean distance given by

$$
D(p \| q)=\sqrt{\int_{-\infty}^{+\infty}[p(x)-q(x)]^{2} d x} .
$$

\section{Features Choice}

A fundamental step in designing (1) or (3) is the choice of the features $\gamma$, extracted by observing the received signal $r(t)$ in a certain observation interval $T$, which are usually more affected by channel conditions.

The first parameter taken into account is the RMS delay spread that captures the temporal dispersion of the energy in a signal. It is defined as

$$
\tau_{\mathrm{rms}}=\sqrt{\frac{\int_{0}^{\infty}\left(t-\tau_{\mathrm{m}}\right)^{2}|r(t)|^{2} d t}{\int_{0}^{\infty}|r(t)|^{2} d t}}
$$

where $\tau_{\mathrm{m}}$ is the mean excess delay given by

$$
\tau_{\mathrm{m}}=\frac{\int_{0}^{\infty} t|r(t)|^{2} d t}{\int_{0}^{\infty}|r(t)|^{2} d t} .
$$

In case of LOS propagation, the strongest path is typically the first one, while in NLOS conditions it is common to have the strongest path preceded by some other smaller echoes resulting in a larger value of the delay-spread. When distributions in (1) are unimodal and $p(\operatorname{LOS})=p(\mathrm{NLOS})^{1}$, then (1) is equivalent to compare $\gamma$ to a suitable threshold $\lambda$ corresponding to the intersection between $p(\gamma \mid$ LOS $)$ and $p(\gamma \mid$ NLOS $)$. In the delayspread case the decision rule takes the form

$$
\text { Decide : }\left\{\begin{array}{ll}
\text { LOS }, & \text { if } \tau_{\mathrm{rms}} \leq \lambda_{\tau} \\
\text { NLOS, } & \text { if } \tau_{\mathrm{rms}}>\lambda_{\tau}
\end{array} .\right.
$$

\footnotetext{
${ }^{1}$ This assumption is usually considered when no a priori information is available.
} 


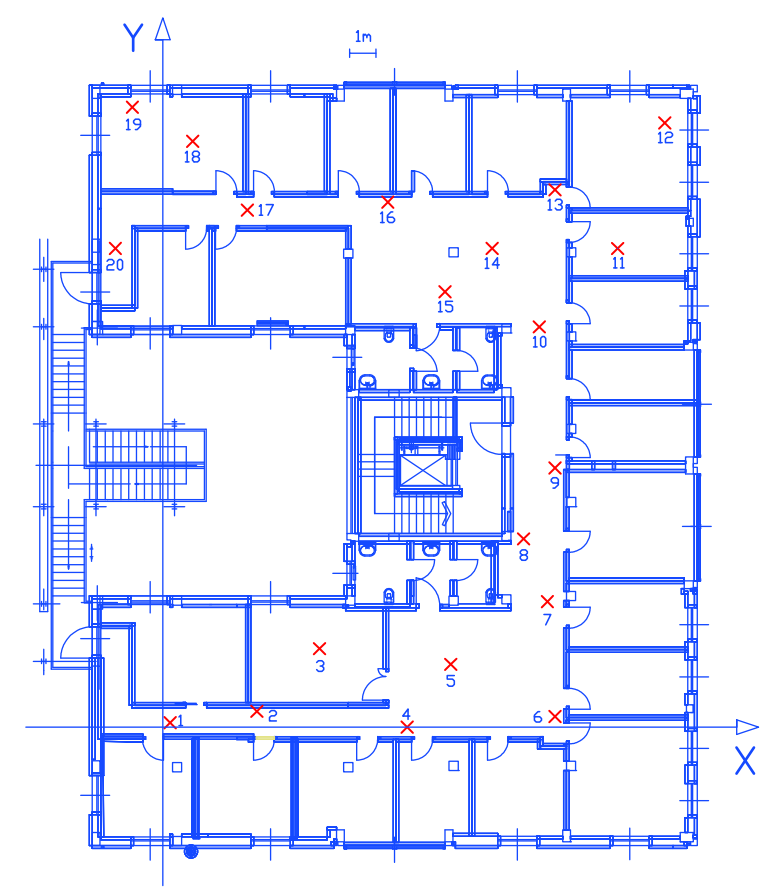

Fig. 2. The experimentation environment at WiLab, University of Bologna, Cesena Campus.

Another parameter taken into account is the kurtosis, defined by

$$
\kappa=\frac{1}{\sigma_{|r|}^{4} T} \int_{T}\left(|r(t)|-\mu_{|r|}\right)^{4} d t
$$

where $\mu_{|r|}=\frac{1}{T} \int_{T}|r(t)| d t$ and $\sigma_{|r|}^{2}=\frac{1}{T} \int_{T}\left(|r(t)|-\mu_{|r|}\right)^{2} d t$. LOS waveforms usually produce a higher value for the kurtosis. For this reason the decision is taken as

$$
\text { Decide }: \begin{cases}\text { NLOS }, & \text { if } \kappa<\lambda_{k} \\ \text { LOS }, & \text { if } \kappa \geq \lambda_{k}\end{cases}
$$

where $\lambda_{k}$ is the threshold value for the detection performed with this scheme. Parameters $\tau_{\text {rms }}$ and $\kappa$ are strongly related to the shape of the waveform. A different feature exploitable for the identification is the energy of the received signal given by

$$
\mathcal{E}_{r}=\int_{T}|r(t)|^{2} d t .
$$

In this case we decide for the NLOS hypothesis if this energy is below a certain threshold due to walls, objects attenuation and reflections, and decide LOS otherwise.

\section{MeAsurements CAMPAign}

Waveforms used for testing the algorithms proposed in previous sections were collected during an extensive measurements campaign at the University of Bologna [20]. The complete measurements database is available in [21].

Two commercial UWB devices, PulsON220 by TimeDomain, were used in this study. These radios operate in a $-10 \mathrm{~dB}$ bandwidth of about $3.2 \mathrm{GHz}$ centered at $4.7 \mathrm{GHz}$ and are equipped with a $2 \mathrm{~dB}$ gain omnidirectional antenna; they

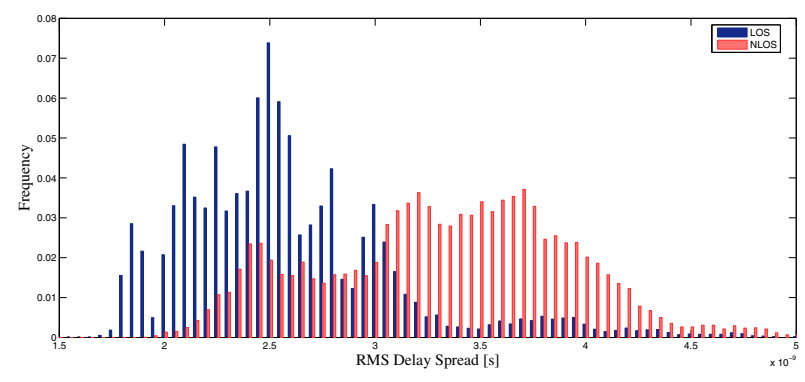

Fig. 3. Example of relative frequency for Delay Spread in LOS and NLOS conditions derived from experimental data

can provide samples of received signals as well as perform ranging estimation using TOA.

The collected waveforms have a length of about $60 \mathrm{~ns}$ and are sampled at about $24.2 \mathrm{GHz}$. In Fig. 1 two typical waveforms relative at LOS and NLOS conditions are presented.

The environment is a typical indoor office with drywall separation of the rooms. In this environment (see Fig. 2) a grid of $N_{p}=20$ positions for the nodes is defined. In this situation we could establish $\left(\begin{array}{c}20 \\ 2\end{array}\right)=190$ theoretical links betweens the devices; however, for the presence of strong attenuations and limited radiated power allowed by FCC masks, the real number of links is significantly lower. In [20] a connectivity matrix that shows which couple of nodes is able to communicate with an acceptable link quality can be found. For each pair of nodes up to 1000 ranging measurements were also taken. During the experiment, there were no moving objects between the two UWB devices. Reflections and attenuations of UWB signals were mainly caused by walls, furniture, or by persons that were standing behind the two devices.

The distances of each node from certain reference points were measured and reported in a computer-aided design (CAD) software to obtain the absolute coordinate respect to a unique reference coordinate system allowing a distinction between link LOS and NLOS.

\section{EXPERIMENTAL RESULTS}

The identification methods have been tested with real waveforms obtained from measurements as described in the previous section. The totality of collected waveforms have been split in two disjoined sets: a training set and a validation set. The former is used for the computation of the reference probability distributions under LOS and NLOS hypotheses and the choice of the thresholds $\lambda_{\tau}, \lambda_{k}, \ldots$, the latter is used for testing the identification algorithms. In Fig. 3 an example of relative frequency distributions for $\tau_{\text {rms }}$ in LOS and NLOS conditions is shown; from that probability distributions are approximated. Each of the two sets contains 500 waveforms collected for each pair of nodes. In this manner we have four disjointed sets; the training set and the validation set for nodes in LOS conditions and the same for nodes in NLOS conditions. In the case of classic identification approaches parameters $\tau_{\text {rms }}$, $\kappa$ and $\mathcal{E}_{r}$ are first computed from the waveform under test; 
TABLE I

Classical IDENTIFICATION APPROACH

\begin{tabular}{|c|c|c|c|c|}
\hline Decision & $\tau_{\text {rms }}$ & $\kappa$ & Joint $\tau_{\text {rms }}-\kappa$ & $\mathcal{E}_{r}$ \\
\hline $\mathcal{H}_{0} \mid \mathcal{H}_{0}$ & 0.77 & 0.74 & 0.88 & 0.96 \\
$\mathcal{H}_{1} \mid \mathcal{H}_{1}$ & 0.79 & 0.77 & 0.79 & 0.87 \\
\hline Error Rate & 0.22 & 0.25 & 0.17 & 0.09 \\
\hline
\end{tabular}

TABLE II

DISTRIBUTION-BASED IDENTIFICATION APPROACH

\begin{tabular}{|c|c|c|c|}
\hline Decision & $\tau_{\text {rms }}$ & $\kappa$ & Joint $\tau_{\text {rms }}-\kappa$ \\
\hline $\mathcal{H}_{0} \mid \mathcal{H}_{0}$ & 0.81 & 0.96 & 0.93 \\
$\mathcal{H}_{1} \mid \mathcal{H}_{1}$ & 0.76 & 0.71 & 0.82 \\
\hline Error Rate & 0.22 & 0.17 & 0.13 \\
\hline
\end{tabular}

then a decision based on (1) or (2) is taken. The percentage of agreements is considered as an indicator of the quality of the identification method. Even though the parameters $\tau_{\text {rms }}$ and $\kappa$ have been already proposed in other papers by considering waveforms drawn from the IEEE 802.15.4a channel model characterized by long channel responses (often $>100 \mathrm{ns)} \mathrm{[9],}$ [10], here the same approach is tested on real data often characterized by shorter channel responses (about $20 \mathrm{~ns}$ ).

In the case of distribution-based identification approach, the observation is taken, instead of on the single waveform, on a certain number of waveforms belonging to the validation set related to the same pair of nodes. Specifically the decision is taken according to (4), having previously built the reference distributions through the training set of waveforms, and again the percentage of agreements is taken as an indicator of the quality.

For the identification based on delay-spread and kurtosis, waveforms have been filtered with a band-pass filter compliant with spectral emission of the devices used during measurements; subsequently they have been normalized to have unitary energy. In this manner only the shape of the signal plays a role in the identification. For the identification based on received energy, waveforms have been clearly only filtered without any other type of processing.

Table I shows the rate of correct and incorrect channel condition identification using classical detecting schemes. As can be noted, $\tau_{\mathrm{rms}}$ and $\kappa$ features give similar results. The third column considers the joint distribution (2) which leads to an improvement in the detection performance. Results related to the energy parameter (11) are reported in column 4 of Table I. Surprising, the performance obtained is remarkable. Probably this result is strictly related to the particular environment under investigation where there is a tight correlation between low signal-to-noise ratio (SNR) and NLOS conditions. This aspect requires further investigations.

Table II refers to the proposed distribution-based approach and shows again the rate of correct and incorrect channel condition identification. For each couple of nodes the distribution is computed using 100 waveforms of the validation set. We can observe how this approach gives in general better results especially when using the kurtosis as parameter. Even in this case using the joint distribution instead of considering a single parameter improves the detection performance.

\section{CONCLUSIONS}

In this paper we have presented some results concerning the identification of the propagation conditions in terms of absence or presence of a line-of-sight link. These results are validated by experimental data collected in an extensive measurement campaign made with FCC-compliant UWB radios. The detection of the LOS/NLOS conditions is performed with a classical binary hypothesis test using root-mean-square delay spread and kurtosis of the received waveforms like features for the identification. We have shown that the two classifiers based on these parameters provide about the same results in terms of correct identifications. The performance can be improved using the two parameters jointly for the test. Furthermore a novel detection scheme which exploits the same features based on distance characterization between probability distributions has been proposed and its performance has been compared with the classic detection schemes.

An important issue that will be investigated in a follow-up paper is the robustness of different identification algorithms to environmental variations.

\section{REFERENCES}

[1] H. Wymeersch, J. Lien, and M. Win, "Cooperative localization in wireless networks," Proceedings of the IEEE, vol. 97, no. 2, pp. 427 -450 , feb. 2009.

[2] S. Gezici, Z. Tian, G. Giannakis, H. Kobayashi, A. Molisch, H. Poor, and Z. Sahinoglu, "Localization via ultra-wideband radios: a look at positioning aspects for future sensor networks," Signal Processing Magazine, IEEE, vol. 22, no. 4, pp. 70-84, July 2005.

[3] Z. Sahinoglu, S. Gezici, and I. Guvenc, Ultra-Wideband Positioning Systems: Theoretical Limits, Ranging Algorithms, and Protocols. Cambridge University Press, 2008.

[4] K. Pahlavan, F. O. Akgul, M. Heidari, A. Hatami, J. M. Elwell, and R. D. Tingley, "Indoor geolocation in the absence of direct path," Wireless Communications, IEEE, vol. 13, no. 6, pp. 50-58, Dec. 2006.

[5] J.-Y. Lee and R. Scholtz, "Ranging in a dense multipath environment using an UWB radio link," Selected Areas in Communications, IEEE Journal on, vol. 20, no. 9, pp. 1677-1683, Dec 2002.

[6] D. Dardari, A. Conti, J. Lien, , and M. Z. Win, "The effect of cooperation on localization systems using UWB experimental data," EURASIP Journal on Advances in Signal Processing, 2008.

[7] D. Dardari, A. Conti, U. Ferner, A. Giorgetti, and M. Win, "Ranging with ultrawide bandwidth signals in multipath environments," Proceedings of the IEEE, vol. 97, no. 2, pp. 404-426, Feb. 2009.

[8] S. Gezici, H. Kobayashi, and H. Poor, "Nonparametric non-line-of-sight identification," in Vehicular Technology Conference, 2003. VTC 2003Fall. 2003 IEEE 58th, vol. 4, Oct. 2003, pp. 2544-2548 Vol.4.

[9] I. Guvenc, C.-C. Chong, and F. Watanabe, "NLOS identification and mitigation for UWB localization systems," in Wireless Communications and Networking Conference, 2007.WCNC 2007. IEEE, March 2007, pp. 1571-1576.

[10] I. Guvenc, C.-C. Chong, F. Watanabe, and H. Inamura, "NLOS identification and weighted least-squares localization for UWB systems using multipath channel statistics," EURASIP Journal on Advances in Signal Processing, 2008.

[11] J. Borras, P. Hatrack, and N. Mandayam, "Decision theoretic framework for NLOS identification," in Vehicular Technology Conference, 1998. VTC 98. 48th IEEE, vol. 2, May 1998, pp. 1583-1587 vol.2.

[12] A. Maali, A. Ouldali, H. Mimoun, and G. Baudoin, "Joint TOA estimation and NLOS identification for UWB localization systems," in Sensor Technologies and Applications, 2009. SENSORCOMM '09. Third International Conference on, June 2009, pp. 212-216. 
[13] M. Heidari, F. Akgul, and K. Pahlavan, "Identification of the absence of direct path in indoor localization systems," in Personal, Indoor and Mobile Radio Communications, 2007. PIMRC 2007. IEEE 18th International Symposium on, Sept. 2007, pp. 1-6.

[14] M. Heidari, F. Akgul, N. Alsindi, and K. Pahlavan, "Neural network assisted identification of the absence of direct path in indoor localization," in Global Telecommunications Conference, 2007. GLOBECOM 'O7. IEEE, Nov. 2007, pp. 387-392.

[15] A. Maali, H. Mimoun, G. Baudoin, and A. Ouldali, "A new low complexity NLOS identification approach based on UWB energy detection," in Radio and Wireless Symposium, 2009. RWS '09. IEEE, Jan. 2009, pp. 675-678.

[16] S. Venkatesh and R. Buehrer, "Non-line-of-sight identification in ultrawideband systems based on received signal statistics," Microwaves, Antennas \& Propagation, IET, vol. 1, no. 6, pp. 1120-1130, Dec. 2007.

[17] K. Yu and Y. Guo, "Non-line-of-sight detection based on TOA and signal strength," in Personal, Indoor and Mobile Radio Communications, 2008. PIMRC 2008. IEEE 19th International Symposium on, Sept. 2008, pp. $1-5$.

[18] — "Statistical NLOS identification based on AOA, TOA, and signal strength," Vehicular Technology, IEEE Transactions on, vol. 58, no. 1, pp. 274-286, Jan. 2009.

[19] S. Maran, W. M. Gifford, H. Wymeersch, and M. Z. Win, "Nonparametric obstruction detection for UWB localization," in Global Telecommunications Conference, 2009. GLOBECOM '09. IEEE, 2009.

[20] D. Dardari and F. Sottile, "WPR.B database: Annex of progress report II on advanced localization and positioning techniques: Data fusion and applications.(eds.)." in Deliverable DB.3 Annex, 216715 Newcom++ NoE, WPR.B, 2009.

[21] [Online]. Available: www.vicewicom.eu 